\title{
Entanglement entropy of an accelerating universe
}

\author{
Pedro F. González-Díaz, ${ }^{1}$ Carmen L. Sigüenza, ${ }^{2}$ and Juan Martín-Carrión ${ }^{3}$ \\ ${ }^{1}$ Colina de los Chopos, Instituto de Física Fundamental, Consejo Superior de Investigaciones Científicas, \\ Serrano 121, 28006 Madrid, Spain \\ ${ }^{2}$ Estación Ecológica de Biocosmología, Pedro de Alvarado 14, 06411-Medellín, Spain, \\ and Colegio Padres Escolapios, Getafe, Madrid, Spain \\ ${ }^{3}$ Telefónica Servicios Audiovisuales, Engineering Department, C/Virgilio 2-Edificio 2, 28223 Pozuelo de Alarcón, Madrid, Spain
}

(Received 6 June 2011; published 3 July 2012)

\begin{abstract}
We have considered the existence of a dependence of the entanglement entropy on the cosmological horizon surface area also in several accelerating models of the current universe both for a quintessence scalar field and for a phantom-energy scenario. It is shown that if a quintessence vacuum cosmic field is considered then, though the case for $w>-1$ satisfies a second law for entanglement entropy, when $w<-1$ such a law is violated. It is finally noted that the entanglement entropy and the distinct formulations of cosmic holography share common future surfaces which are optimal screen for the latter descriptions.
\end{abstract}

DOI: 10.1103/PhysRevD.86.027501

PACS numbers: $98.80 .-\mathrm{k}, 04.70 .-\mathrm{s}$

There are many works in the scientific literature dealing with the connection between entanglement entropy and the gravitational entropy of compact objects or cosmic scenarios, such as black holes [1] and the de Sitter universe [2], in the realm of diverse frameworks, including string theory [3], AdS/CFT correspondence [4], brane worlds [5] or plain gravity [6] (for detailed reviews see Ref. [7]). Actually, entanglement entropy measures a degree of the correlation between subsystems of a given quantum system [8]. In many-body theories quantum entanglement is usually determined when distinct subsystems are spatially separated. Generally speaking, one can say that in an entangled state, the entanglement entropy of the two spatial regions (which can be conventionally separated or correspond to the inside and outside regions of a natural boundary such as an event horizon) must be equal, and this appears to be highly suggestive that the entanglement entropy is proportional to the mutual surface area [9]. Actually, one can show that entanglement of a quantum field between the inside and outside of a horizon can account for the area-proportionality of the black hole [1] and de Sitter space [2] entropies, provided that the field is in its ground state. Two and three-dimensional black holes possess [7] an entanglement entropy whose leading term exactly reproduces the Bekenstein-Hawking entropy, whereas the subleading term behaves logarithmically. Finally, Müller and Lousto [10] developed a cosmological nonaccelerating scenario where future event horizons give rise to well-defined values for an entanglement entropy that is also given in terms of the surface area of the horizon.

All the above results allow us to confidently extrapolate the above horizon area-entanglement entropy proportionality law to any system where quantum entanglement takes place in a cosmic space-time. The results that we are going to get later on are all consistent with that extrapolation. In fact, in this brief report we are going to extend the existence of such a dependence of entanglement entropy on the cosmological horizon surface area also in accelerating models for the current universe both for a quintessence scalar dark-energy field and for a phantom-energy scenario. It will be shown that if an usual quintessence vacuum cosmic field is considered [11], then, though the case for $w>-1$ (note that along this report we shall only consider an equation of state for the Universe with the perfect-fluid form $p=w \rho$, with $p$ the pressure and $\rho$ the energy density) satisfies a second law for entanglement entropy, when $w<-1$ such a law would be violated.

We want to estimate the entanglement entropy that corresponded to a quantum field in an accelerating spacetime which is inexorable endowed with a future event horizon [12]. We shall, therefore, consider the observationally most favored case of a spatially flat FriedmannRobertson-Walker metric whose scale factor is given by [11]

$$
\begin{aligned}
a & =\left[a_{0}^{3(1+w) / 2}+\frac{3}{2} C(1+w)\left(t-t_{0}\right)\right]^{2 /[3(1+w)]} \\
& =T^{2 /[3(1+w)]},
\end{aligned}
$$

where $w=p / \rho$ is the parameter of the equation of state, $a_{0}$ and $t_{0}$ are the initial values of the scale factor and time, and the constant $C$ is given by $C=\sqrt{8 \pi G \mu / 3}$, with $\mu$ an integration constant. We introduce then a conformal time defined by

$$
\eta=\int \frac{d t}{a(t)}=\frac{2 a^{(1+3 w) / 2}}{(1+3 w) C}
$$

so that

$$
a(\eta)=\left[\frac{(1+3 w) \eta C}{2}\right]^{2 /(1+3 w)}
$$


It is worth noting that the conformal time $\eta$ runs from a finite positive constant value

$$
\eta_{0}=-\frac{2 a_{0}^{(1+3 w) / 2}}{C(1+3 w)}
$$

at $t=t_{0}$, to $\eta=0$ as $t \rightarrow \infty$, and runs from that constant value when $w<-1$ first to $\eta=0$, at the big rip [13], to finally diverge as $t \rightarrow \infty$.

We shall consider in what follows cosmic space-times endowed with a future cosmological event horizon, that is the maximal coordinate distance a photon emitted at time $t$ can travel. It can be computed to be given by

$$
r_{H}=a(t) \int_{t}^{\infty} \frac{d t^{\prime}}{a\left(t^{\prime}\right)}=a(\eta) \Delta \eta
$$

In terms of conformal time, the proper event horizon radius becomes

$$
\begin{aligned}
r_{H} & =a(\eta)\left[\eta\left(t_{\text {final }}\right)-\eta(t)\right]=-a(\eta) \eta(t) \\
& =-\frac{2 T}{(1+3 w) C}
\end{aligned}
$$

for $w>-1$, and $r_{H}=+\infty$ for $w<-1$. In order to estimate the entanglement entropy of a phantom universe, instead of the cosmological event horizon, we should use the expression [14]

$$
r_{H}^{\mathrm{ph}}=a(t) \int_{t}^{t_{\mathrm{BR}}} \frac{d t^{\prime}}{a\left(t^{\prime}\right)},
$$

with

$$
t_{\mathrm{BR}}=t_{0}+\frac{2 a_{0}^{-3(|w|-1) / 2}}{3 C(|w|-1)},
$$

the time at which the future big rip singularity takes place. This expression again is given by Eq. (5).

Inserting the conformal time $\eta$, the space-time metric corresponding to a flat accelerating universe can be written as

$$
d s^{2}=T(\eta)^{4 /[3(1+w)]}\left[d \eta^{2}-\left(d x^{2}+d y^{2}+d z^{2}\right)\right],
$$

with parameters $A$ and $D$ as those defined as $C$ and $B$, respectively, in Ref. [10], reading now

$$
A=D \equiv T(\eta)^{2 /(1+3 w)}=\left[\frac{(1+3 w) \eta C}{2}\right]^{2 /(1+3 w)} .
$$

Then, the area spanned by that cosmological event horizon for $w>-1$ can be expressed as [14]

$$
A_{0}=\int_{r=r_{0}} \sqrt{g_{\theta \theta} g_{\phi \phi}} d \theta d \phi=2 \pi a(\eta)^{2} r_{0}^{2},
$$

and for $w<-1$,

$$
A_{0}^{\mathrm{ph}}=2 \pi a(\eta)^{2} r_{0}^{\mathrm{ph} 2}
$$

Using finally Eq. (5), we have

$$
A_{H}=\frac{16 \pi a(\eta)^{5+3 w}}{(1+3 w)^{2} C^{2}},
$$

both for $w>-1$ and $w<-1$.

At first sight, one should then proceed to numerically calculating the entanglement entropy by following the procedure devised in Ref. [10], according to which we expand the used scalar field in spherical harmonics, placing then the system in a spherical lattice with a given lattice spacing $L$. Thus, the entanglement entropy for an accelerating universe could be numerically computed by tracing out one of the two regions in which the event horizon divides the whole space-time. In this way, we could finally obtain the proportionality constant between the entanglement entropy and the horizon surface area. At least in the present case the problem is with the choice of $L$ because different values of $L$ give different results, with an uncertainty that qualitatively overcomes what one would expect to be reasonable. It is for this reason that we shall restrict ourselves in this paper to just assuming the existence of such a proportionality without going to check it by using any numerical estimate.

Extrapolating then the general prescription on entanglement entropy-future horizon surface area to a cosmic curved accelerating space-time with future event horizon [10], and using the geometrical results given above, one can finally show that the entanglement entropy is proportional to the event horizon area also for the case of a flat accelerating universe filled with dark energy endowed with an equation of state $p=w \rho$, both for $w>-1$ and $w<$ -1 ; that is we in general have

$$
S_{\mathrm{Ent}}=\alpha r_{H}^{2},
$$

where $\alpha$ is a constant of order unity. This result can also be readily extended to the other cases being considered in this paper, namely, dynamical dark energy with an extra cosmological constant.

This can be most readily seen by considering the de Sitter universe with a positive cosmological constant $\Lambda$, in which $w=-1$ for the geometry that we have already considered. We then obtain for the entanglement entropy the constant quantity

$$
S_{\mathrm{Ent}}^{d S}=\frac{\alpha^{\prime}}{8 \pi G},
$$

with $\alpha^{\prime}=3 \alpha a_{0}^{2} / \mu$. If we take this entropy to be the origin of the gravitational de Sitter thermal entropy, then the comparison of Eq. (13) with the Gibbons-Hawking formula[15] leads to fixing $\alpha^{\prime}=\pi / \Lambda$. That result looks physically reasonable due to fact that all the lack of universality is completely absorbed by the initial value of the scale factor, that is to say, of the chosen cosmological constant; we actually obtain the quite correct result that $a_{0} \propto 1 / \sqrt{\Lambda}$, so showing the adequacy of our extrapolation 
which we shall consistently assume to take place for all values of the equation of state parameter $w$.

The Gibbons-Hawking entropy corresponds to saturating the entropy bound that describes the upper limit on the gravitational entropy for the bounded de Sitter region. The objection to the existence of such an entropy bound is that as the number of existing field species that can be considered in the theory, $N$, grows, so does the total entropy defined by $S_{T}=N S_{\text {Ent }}$. Thus, even though Bekenstein settled down a universal upper bound on the entropy-toenergy ratio for bounded systems [16], the total entropy would increase with the number of the field species [17]. However, the well-known bound on the entropy-to-energy ratio, first proposed by Bekenstein [16], actually implies [18] the holographic bound on the entropy, and hence that the number of particle species ought to be also bounded [18]. This gives rise to a rather stringent limit on the total entropy of the given bounded region as well.

For general quintessence cosmic fields with $-1 / 3>$ $w>-1$ in the absence of a cosmological constant, from the identification given by Eqs. (11) and (12), we obtain that the entanglement entropy steadily increases from

$$
\frac{\alpha^{\prime} a_{0}^{3(1+w)}}{2 \pi G(1+3 w)^{2}}
$$

for $t=t_{0}$ up to infinity as time $t$ becomes infinity, thus satisfying a second law for that kind of entropy. We note that $S_{\text {Ent }}=\infty$ for any time in the limiting case that $w=$ $-1 / 3$. When we are dealing with a purely dynamical phantom fluid [13] for which $w<-1$, the entanglement entropy as calculated by following the same procedure as for Eq. (12) steadily decreases first from a value also given by Eq. (14) down to zero at the big rip singularity, to increase, thereafter, becoming infinity as time goes to infinity. One still could consider cosmic solutions obtained by adding to the quintessence vacuum a cosmological constant $\Lambda$, that is for a scale factor [19]

$$
\begin{aligned}
a(t)= & \left(\frac{C}{4 B \lambda}\right)^{(1 / 3(1+w))} \times\left[e^{\left(3(1+w) \sqrt{\lambda}\left(t-t_{0}\right) / 2\right)}\right. \\
& \left.-B e^{-\left(3(1+w) \sqrt{\lambda}\left(t-t_{0}\right) / 2\right)}\right]^{(2 / 3(1+w))}
\end{aligned}
$$

where

$$
B=\frac{\sqrt{\lambda+C a_{0}^{-3(1+w)}}-\sqrt{\lambda}}{\sqrt{\lambda+C a_{0}^{-3(1+w)}}+\sqrt{\lambda}}<1,
$$

with $\lambda=\Lambda / 3$. Equation. (15) can describe both a quintessence field with $w>-1$ and a phantom field for $w<-1$. In the latter case the model shows a big rip singularity which is placed at a finite time in the future given by

$$
t=t^{*}=t_{0}-\frac{\ln B}{3(|w|-1) \sqrt{\lambda}} .
$$

One can rather straightforwardly see, however, that even though the entanglement entropy derived from using the above procedure from this analytical solution behaves as expected in the case $w>-1$, as it can be seen for $w=-2 / 3$,

$$
S_{\text {Ent }} \propto\left(1-B e^{-\sqrt{\lambda}\left(t-t_{0}\right)}\right)^{2},
$$

which increases steadily with time down to a finite maximum value constant at $t=\infty$. The phantom case is once again rather problematic as it can be immediately checked for the particular case e.g. $w=-4 / 3$, for which we get a cosmological event horizon which is always placed beyond the big rip singularity at an inexorably infinite and inaccessible distance from any observer (that as to say, no future event horizon may exist in this case).

A rather straightforward connection of the concept of entanglement entropy of an accelerating universe can be traced here to recent developments on cosmic holography, in which there also is at least one hypersurface that divides the space-time into two regions, sometimes at places where expansion converts into contraction. The so-called holographic dark-energy models were first considered by $\mathrm{Li}$ [20] who proposed a holographic law based on black hole physics. Thus, the short distance cutoff was related to the infrared cutoff [20], which he took as the size of the observer-dependent future event horizon making the holographic screen. According to that law, one can write

$$
H^{2}=\frac{8 \pi G \rho}{3}=\frac{c^{2}}{r_{H}^{2}},
$$

where the Hubble parameter is given by $H=\dot{a} / a$, with $a$ being the scale factor of the universe, $\rho$ is the dark-energy density, $r_{H}$ is given by Eq. (4) and interpreted here as the ultimate region whose total energy is taken not to exceed the mass of a black hole with the same size, and $c$ is a numerical parameter of order unity which is defined as

$$
c=-\frac{2}{1+3 w} .
$$

Equation (19) is only valid for $w>-1$ for which case $c>1$. Then, $r_{H}$ should also be regarded to be the proper size of a holographic screen where all information contained in its inner volume is encoded. There also exists another holographic screen in this case. It corresponds to the apparent horizon defined by

$$
\eta_{A}=\frac{2 r_{A}}{1+3 w},
$$

and uncovered by using the Bousso holographic covariant method [21]. It is worth noticing that exactly such a holographic screen also occurs in the phantom regime for 
$w<-1$, provided the parameter $w$ is suitably discretized (see later). In terms of the scale factor we obtain

$$
r_{A}=\frac{a^{(1+3 w) / 2}}{C},
$$

so that the surface area spanned by that surface will be

$$
A_{A}=2 \pi a^{2} r_{A}^{2}=\frac{2 \pi a^{3(1+w)}}{C^{2}}
$$

It follows that the corresponding entanglement entropy should be given by

$$
S_{\mathrm{Ent}}^{A}=\delta a^{3(1+w)},
$$

with $\delta$ as a proportionality constant factor. It is noteworthy that an expression proportional to Eq. (24) can be also derived by directly computing Eq. (5) for $r_{A}$ i.e.,

$$
r_{H}=-\frac{2 a^{3(1+w) / 2}}{C(1+3 w)} \text {. }
$$

As we have already pointed out, the covariant holographic apparent horizon at $\eta_{P}=2 r /(1+3 w)$ also occurs in the phantom region, this time for a value $\eta_{P}=0$ corresponding to the big rip time. This holographic optimal screen can be also obtained by means of the relations [22]

$$
H_{\mathrm{ph}}^{2}=\frac{8 \pi G \rho}{3}=\frac{c^{2}}{r_{\mathrm{br}}^{2}},
$$

where

$$
r_{\mathrm{br}}=a(t) \int_{t}^{t_{\mathrm{br}}} \frac{d t^{\prime}}{a\left(t^{\prime}\right)}
$$

is the proper size of the future horizon as evaluated at the big rip time $t_{\mathrm{br}}$. However, in order for this description to be applicable also after the big rip so that the scale factor be kept real and positive on that region, not all the values of $w$ can be allowed but only those that satisfy the discretization condition

$$
w=-\frac{1}{3}\left(1+\frac{2 n+3}{n+1}\right), \quad n=0,1,2, \ldots
$$

Since the bound on the entropy $S \leq A_{H} / 4$, derived [18] from the entropy-to-energy bound [16] discussed before, strictly corresponds to the holographic bound which is nevertheless currently attributed to 't Hooft, one can straightforwardly see that there must exist a close connection between entropy of entanglement and the covariant definition of holographic entropy [21]. On the other hand, the discretization of the equation of state in case of phan- tom energy arises from the feature that holographic optimal screens making the holographic surfaces, where all the three-dimensional information in the whole space-time manifold is encoded [21] will always be described in the causal development as the latest surface which is expanding [21]. In order to ensure then that a holographic optimal screen has that property it is necessary allowing the causal evolution to reach at least a little region beyond that holographic surface, into a well-defined contracting region. In case of phantom energy the scale factor derived from Eq. (1) for $w<-1$ reads

$$
a=\left[a_{0}^{-3(|w|-1) / 2}-\frac{3}{2} C(|w|-1)\left(t-t_{0}\right)\right]^{-2 /[3(|w|-1)]},
$$

so that all available contracting regions can only be welldefined for those values of the equation of state parameter that satisfy condition (28).

The set of the above results strongly suggests a deep relation between quantum entanglement and cosmic holography as they both are using common hypersurfaces for their respective developments. Such a relation looks reminiscent of a similar more general connection between quantum mechanics and holography, especially in the case of a phantom scenario.

We can sum up the contents of this report by stressing that the entanglement entropy of two distinct models to describe the current accelerating universe has been calculated following the standard accurate procedure put forward by Müller and Lousto [10] and others [23]. Such cosmological models correspond to (i) dark- and phantom-energy quintessential fields, and (ii) dark- and phantom-energy quintessence fields plus a cosmological constant. All the considerations are referred both to $w>-1$ and $w<-1$, estimating the entanglement entropy in each case by using its proportionality to the surface area of the future cosmological event horizon or to the holographic screen placed at the big rip singularity in the case of phantom energy.

Before finishing up, a comment is worth mentioning. It appears that the thermodynamical properties of black holes [24], wormholes [25], ringholes [26], warp drives [27] and de Sitter space [15] can be generalized to the current accelerating cosmology in which the entanglement entropy should be proportional to a gravitational entropy probably given in terms of one fourth the surface area of the future cosmological event horizon.

The authors thank the Estación Ecológica de Biocosmología of Medellín for allowing us to meet other scientists and actors, and have useful conversations in a nice and unassuming working atmosphere. This work was supported by MICINN under Research Project FIS200806332. 
[1] S. Mukohyama, M. Serin and H. Kodama, Phys. Rev. D 55, 7666 (1997).

[2] Y. Iwashita, T. Kobayashi, T. Shiromizu, and H. Yoshima, Phys. Rev. D 74, 064027 (2006).

[3] M. Srednicki, Phys. Rev. Lett. 71, 666 (1993).

[4] C. A. Fuertes-Pascual, UAM-CSIC, Ph.D. thesis, 2009.

[5] D. V. Dursaev, J. High Energy Phys. 09 (2006) 018.

[6] T. Nishioka, S. Ryu, and T. Takayanagi, J. Phys. A 42, 504008 (2009).

[7] M. Cadoni and M. Melis, Entropy 12, 2244 (2010); J. Eisert, M. Cramer, and M. B. Plenio, Rev. Mod. Phys. 82, 277 (2010), and references therein.

[8] R. Brustein, M. B. Einhorn, and A. Yarom, J. High Energy Phys. 01 (2006) 098.

[9] S. Das and S. Shankaranarayanan, J. Phys. Conf. Ser. 68, 012015 (2007).

[10] R. Müller and C. O. Lousto, Phys. Rev. D 52, 4512 (1995).

[11] C. Wetterich, Nucl. Phys. B 302, 668 (1988); J. C. Jackson and M. Dodgson, Mon. Not. R. Astron. Soc. 297, 923 (1998).

[12] T. Banks, arXiv:hep-th/0007146; E. Witten, arXiv:hep-th/ 0106109; X.-G. He, arXiv:astro-ph/0105005.
[13] R. R. Caldwell, Phys. Lett. B 545, 23 (2002); P. F. González-Díaz and C. L. Sigüenza, Nucl. Phys. B 697, 363 (2004); S. M. Carroll, M. Hoffman, and M. Trodden, Phys. Rev. D 68, 023509 (2003).

[14] R. M. Wald, General Relativity (University of Chicago, Chicago, 1984).

[15] G. W. Gibbons and S. W. Hawking, Phys. Rev. D 15, 2738 (1977).

[16] J. D. Bekenstein, Phys. Rev. D 23, 287 (1981).

[17] J. D. Bekenstein, Phys. Rev. D 70, 121502 (2004).

[18] P. F. González-Díaz, Phys. Rev. D 27, 3042 (1983).

[19] P. F. González-Díaz, Phys. Rev. D 68, 084016 (2003).

[20] M. Li, Phys. Lett. B 603, 1 (2004).

[21] R. Bousso, Rev. Mod. Phys. 74, 825 (2002).

[22] P. F. González-Díaz, Gravitation Cosmol. 12, 29 (2006).

[23] L. Bombelli, R. K. Roul, J. Lee, and R. D. Sorkin, Phys. Rev. D 34, 373 (1986).

[24] S. W. Hawking, Commun. Math. Phys. 43, 199 (1975).

[25] P. Martín-Moruno and P. F. González-Díaz, Phys. Rev. D 80, 024007 (2009).

[26] P. F. González-Díaz, Phys. Rev. D 82, 044016 (2010).

[27] P. F. González-Díaz, Phys. Lett. B 653, 129 (2007). 